\title{
AN ASYMPTOTIC ESTIMATE OF BROWNIAN PATH VARIATION
}

PRISCILLA E. GREENWOOD

1. Let $X(t)$ be standard Brownian motion on $[0,1], P$ a finite partition of $[0,1]$, and $P$ a collection of such partitions. Denote $\sum_{t_{i} \in P}\left|X\left(t_{i}\right)-X\left(t_{i-1}\right)\right|^{2}$ by var $(X, P)$. P. Lévy has shown [2] that if $P$ is a fixed increasing sequence of partitions $P_{n}$ which becomes dense in $[0,1]$, then with probability one, $\lim _{n \rightarrow \infty} \operatorname{var}\left(X, P_{n}\right)=1$; whereas if $\rho$ is all finite partitions with probability one, $\sup _{P \in \mathcal{P}} \operatorname{var}(X, P)$ $=\infty$. For fixed $n$, let $P_{n}^{\prime}$ be all partitions containing at most $n$ points. Since almost all sample paths are continuous, $\sup _{P \in \mathcal{P}_{n}^{\prime}} \operatorname{var}(X, P)<\infty$ with probability one. Let $P_{n}$ be all partitions for which the minimum distance between successive points, called the mesh of $P$, is $\geqq 1 / n$. Then $\mathcal{P}_{n}$ is a subset of $\mathcal{P}_{n}^{\prime}$, and for a sample path $X$,

$$
\sup _{P \in \mathscr{P}_{n}} \operatorname{var}(X, P) \leqq \sup _{P \in \mathscr{P}_{n}} \operatorname{var}(X, P) .
$$

A natural question is: How fast does the supremum on the left in (1) increase as $n$ increases? The answer given here is roughly: not faster than $n^{2}$. The possibility of improving the estimate is discussed following the proof.

2. We prove the following theorem:

ThEOREM. Let $X(t)$ be standard Brownian motion on $[0,1]$. Let $\mathcal{P}_{2 n}$ be the collection of partitions of $[0,1]$ having mesh $\geqq 1 / 2 n$. Then

$$
\lim \sup \sup _{P \in \mathbb{P}_{2 n}} \frac{1}{n^{2}} \sum_{t_{i} \in P}\left|X\left(t_{i}\right)-X\left(t_{i-1}\right)\right|^{2} \leqq K
$$

with probability one. $K$ is a constant.

The proof is given in a sequence of lemmas.

LEMma 1. For each continuous Brownian path on $[0,1]$, let

$$
a=\sup _{t \in[0,1]}|X(t)-X(1)|, \quad b=\sup _{t \in[0,1]}|X(t)-X(0)| .
$$

Then for any real $r, P\left\{a^{2}>r\right.$ or $\left.b^{2}>r\right\} \leqq P\left\{4 b^{2}>r\right\}$.

Proof. Let $c=|X(1)-X(0)|$. Clearly $b \geqq c$. Let $t_{1}, t_{2}$ be times at which $a$ and $b$ are attained.

Presented to the Society, August 28, 1968; received by the editors May 20, 1968. 


$$
\begin{aligned}
b & =\left|X(0)-X\left(t_{2}\right)\right| \geqq\left|X(0)-X\left(t_{1}\right)\right| \\
& \geqq\left|X(1)-X\left(t_{1}\right)\right|-|X(1)-X(0)|=a-c .
\end{aligned}
$$

So $a \leqq(b+c) \leqq 2 b$ and the lemma follows.

LEMMA 2. Let $i=1, \cdots, n, r$ real.

$$
\begin{aligned}
P\left\{\sup _{t} \mid X(i / n)\right. & \left.-\left.X(t)\right|^{2}+\left|X(t)-X\left(\frac{i-1}{n}\right)\right|^{2}>r\right\} \\
& \leqq 2 P\left\{\left|X(i / n)-X\left(\frac{i-1}{n}\right)\right|^{2}>r / 8\right\} .
\end{aligned}
$$

Suprema are taken over $[(i-1) / n, i / n]$ unless otherwise stated.

Proof.

$$
\begin{aligned}
& P\left\{\sup _{t}|X(i / n)-X(t)|^{2}+\left|X(t)-X\left(\frac{i-1}{n}\right)\right|^{2}>r\right\} \leqq P\left\{\sup _{t_{1}, t_{2}}\left|X\left(\frac{i}{n}\right)-X\left(t_{1}\right)\right|^{2}+\left|X\left(t_{2}\right)-X\left(\frac{i-1}{n}\right)\right|^{2}>r\right\} \\
& \text { (2) } \quad \leqq P\left\{\sup _{t_{1}}\left|X\left(\frac{i}{n}\right)-X\left(t_{1}\right)\right|^{2}>r / 2\right. \\
&\text { or } \left.\sup _{t_{2}}\left|X\left(t_{2}\right)-X\left(\frac{i-1}{n}\right)\right|^{2}>r / 2\right\} \\
& \quad \leqq P\left\{\sup _{i_{2}}\left|X\left(t_{2}\right)-X\left(\frac{i-1}{n}\right)\right|^{2}>r / 8\right\},
\end{aligned}
$$

by Lemma 1 , where the interval $[(i-1) / n, i / n]$ replaces $[0,1]$. The distribution of $|X(t)-X((i-1) / n)|$ for $t$ in $[(i-1) / n, i / n]$ is the same as that of $|X(t)|$ for $t \in[0,1 / n]$, where $X(0)=0$. Taking supremum and infimum over $[0,1 / n]$ and using a familiar equality for Brownian motion [1, p. 171],

$$
\begin{aligned}
P\left\{\sup _{t}|X(t)|>c\right\} & \leqq P\left\{\inf _{t} X(t)<-c\right\}+P\left\{\sup _{t} X(t)>c\right\} \\
& =2 P\{X(1 / n)<-c\}+2 P\{X(1 / n)>c\} \\
& =2 P\{|X(1 / n)|>c\}
\end{aligned}
$$

it follows that (2) is $\leqq 2 P\left\{|X(i / n)-X((i-1) / n)|^{2}>r / 8\right\}$.

LEMMA 3. If $F$ and $G$ are distribution functions such that $1-F(x)$ $\leqq 2(1-G(x))$ for all real $x$, then $1-F^{n^{*}}(x) \leqq 2^{n}\left(1-G^{n^{*}}(x)\right)$. 
In fact, a better inequality of this type can be proved, but it does not improve our estimate.

Proof. Assume $1-F^{n-1^{*}} \leqq 2^{n-1}\left(1-G^{n-1^{*}}\right)$. Then

$$
\begin{aligned}
1-F^{n} & =1+\left(1-F^{n-1^{*}}\right) * F-F \\
& \leqq 1-F+2^{n-1}\left(1-G^{n-1^{*}}\right) * F \\
& =1-F+2^{n-1} F-2^{n-1} G^{n-1^{*}}+2^{n-1}(1-F) * G^{n-1^{*}} \\
& \leqq 1+\left(2^{n-1}-1\right) F-2^{n-1} G^{n-1^{*}}+2^{n}(1-G) * G^{n-1^{*}} \\
& =1+\left(2^{n-1}-1\right) F+2^{n-1} G^{n-1^{*}}-2^{n} G^{n^{*}} \leqq 2^{n}\left(1-G^{n^{*}}\right) .
\end{aligned}
$$

The following simple inequality is stated as a lemma for ease of reference.

LEMmA 4. For any sequence of real numbers $\left\{a_{i}\right\}, i=0, \cdots, n$, $\left(a_{n}-a_{0}\right)^{2} \leqq n^{2} \sum_{i=1}^{n}\left(a_{i}-a_{i-1}\right)^{2}$.

LEMma 5. Let $P_{n}$ be the collection of all finite partitions $\left\{0=t_{0}<t_{1}<\cdots<t_{m}=1\right\}$ of $[0,1]$ having mesh $\geqq 1 / n$. Then

$$
P\left\{\sup _{P \in \mathbb{P}_{n}} \operatorname{var}(X, P)>r\right\}
$$

$$
\leqq 2^{n} P\left\{8 n^{2} \sum_{i=1}^{n}\left|X\left(\frac{i}{n}\right)-X\left(\frac{i-1}{n}\right)\right|^{2}>r\right\} \text {. }
$$

Proof. Between successive points of a partition $P \in \mathcal{P}_{n}$ fall $m$ points of the form $i / n, 0 \leqq m \leqq n$, say $t_{j-1}<(i+1) / n<\cdots<(i+m) / n<t_{j}$. By Lemma 4,

$$
\begin{aligned}
& \left|X\left(t_{j-1}\right)-X\left(t_{j}\right)\right| \\
& \qquad m^{2}\left[\left|X\left(t_{j}\right)-X\left(\frac{i+m}{n}\right)\right|^{2}+\cdots\right. \\
& \left.\quad+\left|X\left(\frac{i+2}{n}\right)-X\left(\frac{i+1}{n}\right)\right|^{2}+\left|X\left(\frac{i}{n}\right)-X\left(t_{j-1}\right)\right|^{2}\right] .
\end{aligned}
$$

Summing over $t_{j} \in P$ and collecting the terms pertaining to each interval $[(i-1) / n, i / n]$, we have

$$
\operatorname{var}(X, P) \leqq n^{2} \sum_{i=1}^{n}\left|X\left(\frac{i}{n}\right)-X\left(t_{i}\right)\right|^{2}+\left|X\left(t_{i}\right)-X\left(\frac{i-1}{n}\right)\right|^{2}
$$

$$
\operatorname{var}(X, P) \leqq n^{2} \sum_{i=1}^{n}\left|X\left(\frac{i}{n}\right)-X\left(\frac{i-1}{n}\right)\right|^{2}
$$

The upper expression appears in the sum for $i$ such that there is a 
point $t_{i}$ of $P$ in $((i-1) / n, i / n)$; otherwise the lower expression appears.

We only increase the supremum in (3) if we replace $\rho_{n}$ by the larger collection of partitions having at most one point in each interval $[(i-1) / n, i / n]$. The supremum of the right side of (4) over all such partitions is the sum of the suprema obtained by varying each $t_{i}$ over $[(i-1) / n, i / n]$, i.e.

$$
\begin{aligned}
P\left\{\sup _{P \in \mathbb{P}_{n}} \operatorname{var}(X, P)>r\right\} & \\
\leqq & \left(n^{2} \sum_{i=1}^{n} \sup _{t_{i} \in[(i-1) / n, i / n]}\left|X\left(\frac{i}{n}\right)-X\left(t_{i}\right)\right|^{2}\right. \\
& \left.+\left|X\left(t_{i}\right)-X\left(\frac{i-1}{n}\right)\right|^{2}>r\right\} \\
& =P\left\{n^{2} \sum_{i=1}^{n} Y_{i}>r\right\} .
\end{aligned}
$$

The equality (5) defines independent random variables $Y_{i}, i$ $=1, \cdots, n$, with a common distribution; call it $F$. Lemma 2 says that $1-F(x) \leqq 2(1-G(x))$, where $G$ is the common distribution of the independent random variables $8|(X(i / n)-X(i-1) / n)|^{2}$. Lemma 3 compares the distributions of the corresponding sums:

(6) $P\left\{n^{2} \sum_{i=1}^{n} Y_{i}>r\right\} \leqq 2^{n} P\left\{8 n^{2} \sum_{i=1}^{n}\left|X\left(\frac{i}{n}\right)-X\left(\frac{i-1}{n}\right)\right|^{2}>r\right\}$.

Combining (5) and (6) we have Lemma 5.

Lemma 6. For a positive function $g(n)$,

$$
P\left\{n \sum_{i=1}^{2 n}\left|X\left(\frac{i}{2 n}\right)-X\left(\frac{i-1}{2 n}\right)\right|^{2}>g(n)\right\}=\frac{1}{\Gamma(n)} \int_{g(n)}^{\infty} t^{n-1} e^{-t} d t .
$$

Proof. In order to simplify the form of the resulting integral we consider the $2 n$ independent normally distributed variables $n^{1 / 2}(X(i / 2 n)-X((i-1) / 2 n))$, which have mean 0 and variance $1 / 2$. The distribution of the sum is, then, the indicated $\chi^{2}$ distribution $[1$, p. 47].

LEMMA 7.

$$
\sum_{n=1}^{\infty} \frac{2^{2 n}}{\Gamma(n)} \int_{0}^{\infty} t^{n-1} e^{-t} d t<\infty \quad \text { if } g=K n,
$$

where $K$ is an appropriate constant. 
Proof. Integration-by-parts of the $n$th term gives

$$
\begin{aligned}
& \frac{2^{2 n}}{\Gamma(n)} e^{-g} g^{n-1}\left[1+\frac{n-1}{g}+\cdots+\frac{(n-1) !}{g^{n-1}}\right] \\
& \quad \leqq 2^{2 n} e^{-g} g^{n-1} n^{2} / n ! \sim c \cdot 2^{2 n} e^{-g} g^{n-1} n^{2} / n^{n+1 / 2} e^{-n} \\
& \quad=c \exp [n(1+\log 4)-g+(n-1) \log g-(n-3 / 2) \log n] .
\end{aligned}
$$

Let $g(n)=n\left(1+\log 4+K_{2}\right)=n\left(K_{1}+K_{2}\right)$. Then (7) becomes

$$
\begin{array}{r}
\exp \left[-n K_{2}+n \log n+n \log \left(K_{1}+K_{2}\right)-\log \left[n\left(K_{1}+K_{2}\right)\right]-(n-3 / 2) \log n\right] \\
=\exp \left[-n\left[K_{2}-\log \left(K_{1}+K_{2}\right)\right]+\frac{1}{2} \log n-\log \left(K_{1}+K_{2}\right)\right]
\end{array}
$$

which is summable if and only if $K_{2}>\log \left(K_{1}+K_{2}\right)$.

Proof of Theorem. Lemmas 5 and 6 give

$$
\begin{aligned}
P\left\{\sup _{P \in \mathscr{P}_{2 n}} \operatorname{var}(X, P)>K n^{2}\right\} & \leqq 2^{2 n} P\left\{8 \sum_{i=1}^{2 n}\left|X\left(\frac{i}{2 n}\right)-X\left(\frac{i-1}{2 n}\right)\right|^{2}>K\right\} \\
& =\frac{2^{2 n}}{\Gamma(n)} \int_{n K}^{\infty} t^{n-1} e^{-t} d t,
\end{aligned}
$$

which is summable for a constant $K$ computed in Lemma 7. By the first Borel-Cantelli lemma, the event $\left\{\sup _{P \in \mathscr{P}_{2 n}} \operatorname{var}(X, P)>K n^{2}\right\}$ happens only finitely of ten with probability one, i.e. $\lim \sup _{n \rightarrow \infty}\left(1 / n^{2}\right)$ $\sup _{P \in \mathcal{P}_{2 n}} \operatorname{var}(X, P) \leqq K$ with probability one.

3. Remaining questions. The inequality (4) in Lemma 5 appears wasteful since the factor $m$, which we replaced by $n$, need be no larger than the maximum length of any consecutive sequence of points $i / 2 n$ on which $X$ is monotonic, i.e.

$$
X\left(\frac{i}{2 n}\right)<X\left(\frac{i+1}{2 n}\right)<\cdots, \text { or } X\left(\frac{i}{2 n}\right)>X\left(\frac{i+1}{2 n}\right)>\cdots .
$$

A long sequence of monotonicity should be relatively improbable. It appears doubtful, however, that the estimate can be improved by consideration of such sequences.

Another remaining question is whether the idea of Lévy's proof $[2$, p. 516] can be made to yield a lower bound for the rate of increase of $\sup _{P \in \mathcal{P}_{n}} \operatorname{var}(X, P)$.

\section{REFERENCES}

1. W. Feller, An introduction to probability theory and its applications, Vol. II, Wiley, New York, 1966.

2. P. Lévy, Le mouvement brownien plan, Amer. J. Math. 62 (1940), 487-550.

University of British Columbia 